\title{
3D Image Matching Using a Finite Element Based Elastic Deformation Model
}

\author{
Matthieu Ferrant ${ }^{1,2}$, Simon K. Warfield ${ }^{1}$, Charles R.G. Guttmann ${ }^{1}$, \\ Robert V. Mulkern ${ }^{1,3}$, Ferenc A. Jolesz ${ }^{1}$, and Ron Kikinis ${ }^{1}$ \\ 1 Radiology, Brigham and Womens Hospital, Harvard Medical School, Boston, USA \\ 2 Telecommunications Laboratory, Université catholique de Louvain, Belgium \\ 3 Radiology, Children's Hospital, Boston, USA \\ \{ferrant, warfield, guttmann, jolesz, kikinis\}@bwh.harvard.edu
}

\begin{abstract}
We present a new approach for the computation of the deformation field between three dimensional (3D) images. The deformation field minimizes the sum of the squared differences between the images to be matched and is constrained by the physical properties of the different objects represented by the image. The objects are modeled as elastic bodies. Compared to optical flow methods, this approach distinguishes itself by three main characteristics: it can account for the actual physical properties of the objects to be deformed, it can provide us with physical properties of the deformed objects (i.e. stress tensors), and computes a global solution to the deformation instead of a set of local solutions. This latter characteristic is achieved through a finite-element based scheme. The finite element approach requires the different objects in the images to be meshed. Therefore, a tetrahedral mesh generator using a precomputed case table and specifically suited for segmented images has been developed. Preliminary experiments on simulated data as well as on medical data have been carried out successfully. Tested medical applications included muscle exercise imaging and ventricular deformation in multiple sclerosis.
\end{abstract}

\section{Introduction}

During the last decade, physically realistic models for surgical planning and image registration have gained increased attention in the medical imaging community. The reason for this is that purely image-based statistical methods do not take into account the physical properties of the objects depicted in the image and often cannot predict any changes in the image.

Different imaged objects have very different properties and react in a way defined by their material characteristics (e.g. bone and soft tissues have very different behaviors when submitted to equivalent stresses). Therefore, we believe that using a model incorporating the object's physical characteristics can improve the accuracy of a deformable model significantly.

The discretization of deformation problems using the Finite Element (FE) Method and elastic bodies is becoming more and more popular for various applications such as surgical simulation and surgical planning [1,2,3]. This method, in conjunction with an elastic deformation model, is often chosen for its reliable 
behavior and accuracy as compared to simpler analogies such as mass-spring models and others $[4,5]$.

Previous work for recovering image deformation is mainly based on local image structure [6,7]. These methods compute a deformation field between images simultaneously minimizing a local similarity measure and satisfying some kind of arbitrarily chosen smoothness constraint. They are often referred to as optical flow (OF) methods. Later, the image registration community proposed physical deformation models to constrain the deformation field using elastic [8,9] or even viscous fluid deformation models $[10,11]$. It is only recently that biomechanical models have been explicitly proposed to constrain the deformation of images $[12,13]$. Currently, the drawback of the latter methods is that they either require user intervention, or another means to compute the forces applied to the model. Another drawback is that these methods have only been applied to $2 \mathrm{D}$ images thereby limiting the clinical utility and the possibility to efficiently assess the accuracy of the method.

We propose a new integrated approach that implicitly computes the forces applied to the 3D model by constraining the deformation field to satisfy both the elasticity model and the local image similarity criterion. This is achieved by embedding an image similarity constraint on the deformation field into the minimization scheme that leads to the constitutive equations of the deformation model. The equations are discretized using the finite element method.

We apply this method to synthetic 3D images as well as to sequences of arm exercise and enlarging ventricles in 3D brain MRI. In these applications, the deformations happen over time and are, at least in part, intrinsically due to small biomechanical deformations for which our elastic model is very well suited.

\section{Theory}

\subsection{Mathematical Formulation of the Problem}

We formulate the elastic matching of two images as an energy minimization procedure, where the energy comprises a term modeling the physical behavior of the object to be deformed and another term driving the model so as to match both images. The matching criterion between both images is modeled as the minimization of the sum of the squared differences between both images.

Assuming a linear elastic continuum with no initial stresses or strains, the potential energy of an elastic body submitted to externally applied forces can be expressed as [14]:

$$
E=\int_{\Omega} \sigma^{t} \epsilon d \Omega+\int_{\Omega} F u d \Omega
$$

where $F$ is the vector representing the forces applied to the elastic body (forces per unit volume, surface forces or forces concentrated at the nodes), $u$ the displacement vector, and $\Omega$ the body on which one is working. $\epsilon$ is the strain vector, defined as

$$
\epsilon=\left(\frac{\partial u}{\partial x}, \frac{\partial u}{\partial y}, \frac{\partial u}{\partial z}, \frac{\partial u}{\partial x}+\frac{\partial u}{\partial y}, \frac{\partial u}{\partial y}+\frac{\partial u}{\partial z}, \frac{\partial u}{\partial x}+\frac{\partial u}{\partial z}\right)^{t}=L u
$$


and $\sigma$ the stress vector, linked to the strain vector by the material's constitutive equations. In the case of linear elasticity, with no initial stresses or strains, this relation is described as

$$
\sigma=\left(\sigma_{x}, \sigma_{y}, \sigma_{z}, \tau_{x y}, \tau_{y z}, \tau_{x z}\right)^{t}=D \epsilon
$$

where $D$ is the elasticity matrix characterizing the material's properties [14].

The external forces $F$ can be computed as a classical optical flow field between the images to be matched, providing us with a semi-implicit method where the optical flow field would be an initial estimate of the deformation field being regularized by the elastic model. The estimates can then be iteratively refined until an equilibrium is reached.

To avoid the separate computation of the forces $F$, the elastic deformation, and the matching criterion, we propose to directly compute a deformation field that readily satisfies both the elasticity constraint and a local image similarity constraint between the images to be matched $\left(I_{1}\right.$ and $\left.I_{2}\right)$. Hence, the total energy to be minimized is expressed as:

$$
E=\int_{\Omega} \sigma^{t} \epsilon d \Omega+\int_{\Omega}\left(I_{1}(x+u(x))-I_{2}(x)\right)^{2} d \Omega
$$

Assuming that the deformation field is small and the variation of $I_{1}$ smooth, the first order Taylor expansion of $I_{1}(x+u(x))$ can be expressed as

$$
I_{1}(x+u(x)) \cong I_{1}(x)+\left\langle\nabla I_{1}(x), u(x)\right\rangle
$$

Using the material's constitutive equation (3) and (5), equation (4) becomes (the depencies to $x$ are omitted in further developments to clarify the equations) :

$$
E=\int_{\Omega} \epsilon^{t} D \epsilon d \Omega+\int_{\Omega}\left(I_{1}-I_{2}\right)^{2}-2\left(I_{1}-I_{2}\right) \nabla I_{1} u+u^{t} \nabla I_{1}^{t} \nabla I_{1} u d \Omega
$$

Within a finite element discretization framework, an elastic body can be approximated as an assemblage of discrete finite elements interconnected at nodal points on the element boundaries. The displacements are a function of the displacement at the element's nodal points weighted by the element's shape functions $N_{i}^{e l}(x)(7)$.

$$
u(x)=\sum_{i=1}^{4} N_{i}^{e l}(x) u_{i}^{e l}(x)
$$

The elements we use are tetrahedra, with linear interpolation of the displacement field. Hence, the shape function of node $i$ of tetrahedron $e l$ is defined as follows:

$$
N_{i}^{e l}=\frac{1}{6 V^{e l}}\left(a_{i}^{e l}+b_{i}^{e l} x+c_{i}^{e l} y+d_{i}^{e l} z\right)
$$

The computation of the volume of the element $V^{e l}$ and the other constants is detailed in [14]. For every node $i$ of each element $e l$, we define the matrix 
$B_{i}^{e l}=L_{i} N_{i}^{e l}$. The function to be minimized at every node $i$ of each element $e l$ can thus be expressed as :

$$
\begin{aligned}
E\left(u_{i}^{e l}\right) & =\int_{\Omega} \sum_{j=1}^{4} u_{i}^{e{ }^{t}} B_{i}^{e l}{ }^{t} D B_{j}^{e l} u_{j}^{e l} d \Omega \\
& +\int_{\Omega}\left(I_{1}-I_{2}\right)^{2}-2\left(I_{1}-I_{2}\right) \nabla I_{1} N_{i}^{e l} u_{i}^{e l} d \Omega \\
& +\int_{\Omega} \sum_{j=1}^{4} u_{i}^{e l}{ }^{t} N_{i}^{e l} \nabla I_{1}^{t} \nabla I_{1} N_{j}^{e l} u_{j}^{e l} d \Omega
\end{aligned}
$$

We seek the minimum of this function by solving for $\frac{d E\left(u_{i}^{e l}\right)}{d u_{i}^{e l}}=0$. Equation (9) then becomes :

$$
\int_{\Omega} \sum_{j=1}^{4}\left(B_{i}^{e l}{ }^{t} D B_{j}^{e l}+N_{i}^{e l} \nabla I_{1}^{t} \nabla I_{1} N_{j}^{e l}\right) u_{j}^{e l} d \Omega=\int_{\Omega}\left(I_{1}-I_{2}\right) \nabla I_{1} N_{i}^{e l} d \Omega
$$

This last expression can be written as a matrix system for each finite element:

$$
\left(K^{e l}+G^{e l}\right) u^{e l}=F^{e l}
$$

Matrices $K^{e l}, G^{e l}$ and $F^{e l}$ are defined as follows: $K_{i, j}^{e l}=\int_{\Omega} B_{i}^{e l}{ }^{t} D B_{j}^{e l} d \Omega$, $G_{i, j}^{e l}=\int_{\Omega} N_{i}^{e l} \nabla I_{1}^{t} \nabla I_{1} N_{j}^{e l} d \Omega, F_{j}^{e l}=\int_{\Omega}\left(I_{1}-I_{2}\right) \nabla I_{1} N_{i}^{e l} d \Omega$; where every element $i, j$ refers to pairs of nodes of the element $e l$ ( $i$ and $j$ range from 1 to 4 ). $K_{i, j}^{e l}$ and $G_{i, j}^{e l}$ are 3 by 3 matrices, $F_{j}^{e l}$ is a 3 by 1 vector. The 12 by 12 matrices $K^{e l}$ and $G^{e l}$, and the vector $F^{e l}$ are computed for each element and are then assembled in a global system the solution of which will provide us with the deformation field corresponding to the global minimum of the total energy.

\subsection{Tetrahedral Mesh Generation}

Within the finite element framework, objects need to be meshed, i.e. divided into finite elements. We have chosen tetrahedral elements for their simplicity in terms of shape functions and data structure. Most available packages do not allow meshing of multiple objects [17],[19], and are often designed for regular objects, which is not the case for labeled medical data. Therefore, we have developed our own tetrahedral mesh generator, specifically suited for labeled 3D volumes.

The labeled 3D image is first divided into cubes of a given size, which are further divided into 5 tetrahedra with an alternating pattern so as to avoid diagonal crossings on the shared quadrilateral faces of neighboring cubes. For each tetrahedron, the image labels at its nodes are checked. A case table draws the elements to be added to the mesh. If all 4 nodes have non-object labels, no tetrahedron is added to the mesh. If all nodes have an object label, the tetrahedron is added to the mesh as is. If the tetrahedron lies across two objects (i.e. all nodes do not have the same label), the subdivision of the original tetrahedron is looked 

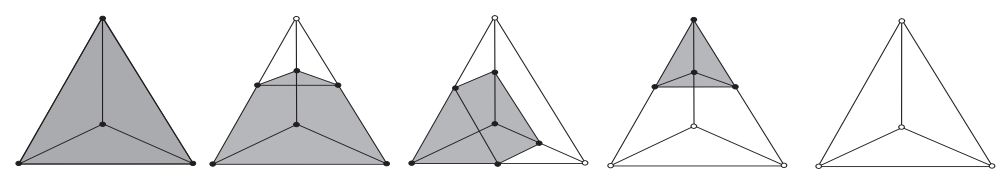

Fig. 1. Different tetrahedral cases depicted from left to right. Case 1: all nodes belong to structure; case 2: 3 nodes belong to structure; case 3: 2 nodes belong to structure; case 4: 1 node belongs to structure; case 5: no nodes belong to structure.

up in the case table. Figure 1 shows the 5 basic cases. There are actually 16 cases, but the remaining cases are symmetric to cases 2, 3 and 4 . The resolution of the mesh can easily be adapted by varying the tetrahedra's sizes. The resulting prisms are divided into tetrahedra using Nielson's index connexion rule [15] so as to avoid edge crossings on the quadrilateral faces shared by neighboring prisms. The mesh structure is built such that for images containing multiple objects, a

a)
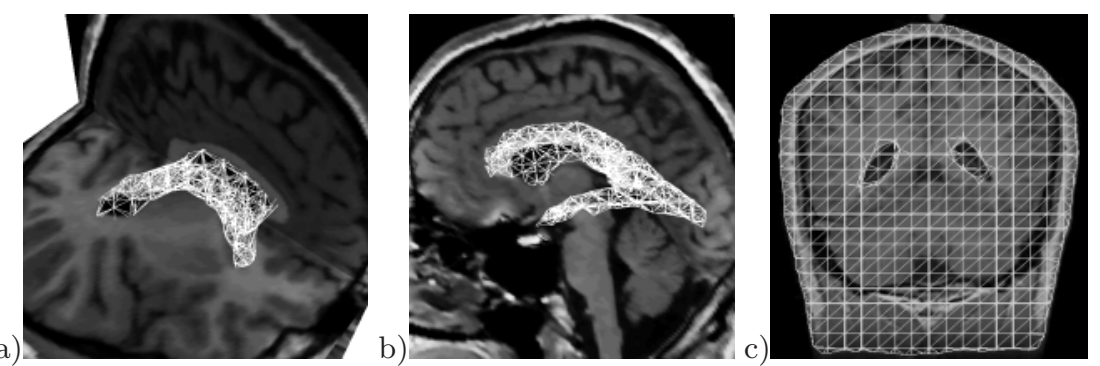

Fig. 2. a) and b) 3D rendering of the wireframe tetrahedral mesh of lateral ventricles. c) coronal cut through tetrahedral mesh of head and lateral ventricles.

fully connected and consistent tetrahedral mesh is obtained with for every cell, a given label corresponding to the object the cell belongs to. Therefore, different biomechanical properties can easily be assigned to the different cells or objects composing the mesh.

\subsection{Material Properties}

An isotropic linear elastic material is characterized by two parameters: Young's elasticity modulus $E$ and Poisson's ratio $\nu$ [14]. They determine the elastic behavior of the object to be deformed and are related to the Lamé constants $\lambda$ and $\mu$ by the following relations :

$$
E=\frac{\mu(2 \mu+3 \lambda)}{\mu+\lambda} \quad \nu=\frac{\lambda}{2(\lambda+\mu)}
$$

where $E$ relates tension in the object and its stretch in the longitudinal direction, and $\nu$ is the ratio of the lateral contraction to longitudinal stretch.

The choice of these values is of course critical to the reliability of a physics based deformation model. Their determination has not been addressed very consistently in the literature as the coefficients used often differ significantly from 
study to study and do not always include the physical units of the values. Recently, Hagemann et. al. [13] published a comparative study of brain elasticity coefficients proposed by different authors, and came to the conclusion that for their application, the only comparable and meaningful values presented by other authors are the ratios of the coefficients. This has also been our choice for all the presented experiments.

\section{Experiments}

We have implemented our own FE algorithm. The assembly and solving of the linear matrix system has been parallelized using the PETSc library [16]. The matching algorithm, using a mesh with approximately hundred thousand tetrahedra, only takes a few minutes on a parallel machine with 20 Ultra Sparc II $250 \mathrm{Mhz}$ CPUs. The size of the edges of the tetrahedra was approximately $5 \mathrm{~mm}$, so as to have a good compromise between capture range and local precision. The $3 \mathrm{D}$ visualization module has been programmed using The Visualization Toolkit library [17].

An experiment with a synthetic image was carried out to verify the plausibility of our model and to show the advantage, for medical imaging applications, of our matching using an elastic model instead of just considering local image structure with a smoothness constraint (Optical Flow methods). To demonstrate the applicability of our method, we also chose two experiments with medical data.

\subsection{Growing Sphere Experiment}

We have applied the algorithm to a synthetic sequence of two spheres (gray regions) centered at the same location with a radius of 15 and 17 pixels. On figure 3, one can observe that the deformation field yielded by $\mathrm{OF}$ is located only at the voxels where the difference between both images is non-zero, while the FE elastic deformation algorithm propagates the deformation all along the surrounding elastic body (which in this experiment was stiffer and had a larger Young modulus than the sphere itself).

a)

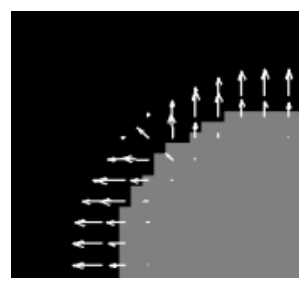

b)

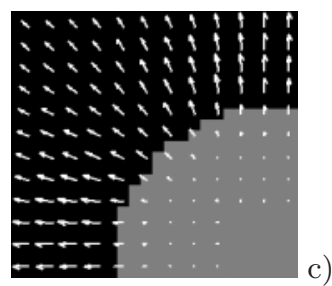

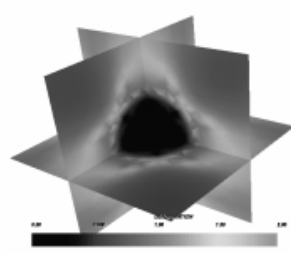

Fig. 3. Growing sphere. a) and b): close-ups of 2D cuts through 3D image with a) classical OF, and b) FE matching deformation fields overlayed, c) and 3D orthogonal cuts through the FE mesh with intensity coding of the displacement field. The displacement field is mainly located at the boundaries of the sphere and is propagated through the surrounding elastic medium. 


\subsection{Arm Data}

The next example studies the deformation occurring when a muscle of the arm is exercised by a two finger flexion [18]. Exercise causes a muscle on the left part of the image to expand. The aim of this exercise related muscle deformation experiment was to characterize the physical change happening during exercise, by comparing both images when they are aligned together.
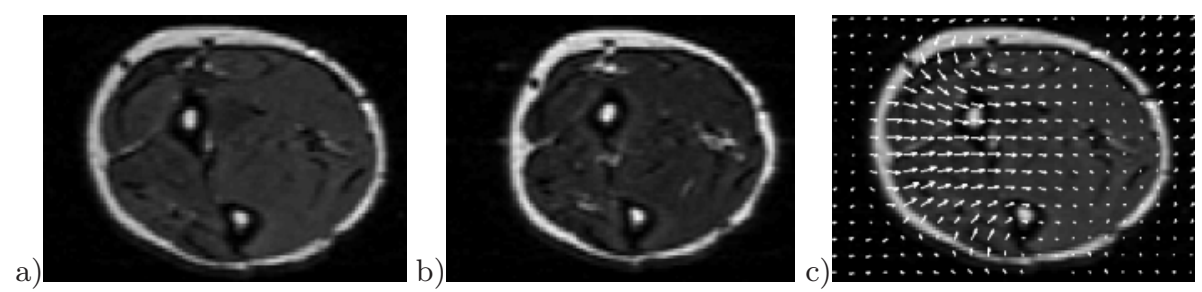

Fig. 4. Arm exercise. Slice of 3D MR dataset a) at exercise, b) at rest, c) deformation field overlayed on exercise slice

In this experiment, the Young modulus of the tissue has been set to $2 \mathrm{kPa}$ (and $\nu$ to 0.3 ), and was constant over the whole arm volume. Future enhancements will include different coefficients for the bones and the skin. The results of Figure 4 confirm that the muscular exercise manifests itself essentially on the left part in the image, where the displacement field is the most important.

\subsection{Ventricular Matching}

In this experiment, the ventricles and the intra-cranial cavity of an MS patient have been segmented at two different time points (approx. 3 years apart) from 3D T2 weighted MR images. During that period, significant enlargement of the ventricles occurred. The matching of these time points allows us to observe the change in shape of the brain and ventricles $(E=3 k P a, \nu=0.4)$.

a)

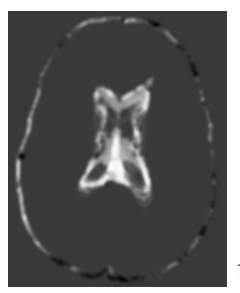

b)

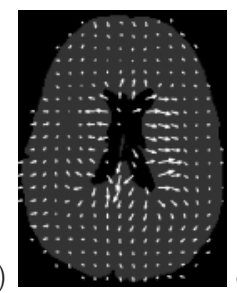

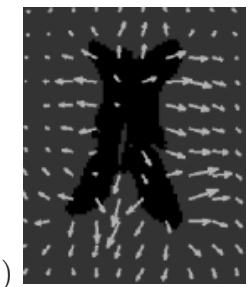

Fig. 5. Enlarging ventricles.a) slice of difference between segmented images at both time points (gray means no difference), b)deformation field superimposed on same image at the first time point. c) close-up 


\section{Conclusions and Perspectives}

We have presented a new, physics-based deformable model for tracking physical deformations using image matching. The model results from the minimization of a deformation field simultaneously satisfying the constraints of an elastic body and a local image similarity measure. The model provides us with a physically realistic deformation field and also allows us to inspect the characteristics of the deformed objects. This can be very useful for the inspection of stresses induced by the deformation of certain objects on their surroundings. For example, the model could be used to predict deformations consequent to the growth of a tumor, to predict brain shift during neurosurgy, etc.

In the experiments we presented, the objects were considered to be homogeneous elastic bodies. Further improvements of the algorithm include the assignment of different elasticities to the different objects represented in the image. This will require a preliminary segmentation of the objects to be deformed so as to be able to set appropriate elasticity coefficients to every cell of the mesh. Also, the anisotropy of certain tissues could be included into the model by modifying the elasticity matrix $D$ appropriately.

\section{Acknowledgements}

M. Ferrant is working towards a Ph.D. degree with a grant from the Belgian FRIA. This investigation was also supported in part by a grant from the National Multiple Sclerosis Society (SW) and by the Neuroimage Analysis Center (NIH P41 RR132118-01), Core Segmentation Tools for Computer Assisted Surgery (NIH R01 RR11747-01A), and MR Guided Therapy (NIH P01 CA67165-03). The authors would also like to thank Dr. Xingchang Wei for providing us with the segmented images for the ventricular matching experiment.

\section{References}

1. H. Delinguette. Toward Realistic Soft-tissue Modeling in Medical Simulation. Proceedings of the IEEE, 86(3):512-523, march 1998. 202

2. D.D. Paulsen, M.I. Miga, F.E. Kennedy, P.J. Hoopes, A. Hartov, and D.W. Roberts. A Computational Model for Tracking Subsurface Tissue Deformation During Stereotactic Neurosurgery. IEEE Transactions on Biomedical Engineering, 46(2):213-225, february 1999. 202

3. O. Skrinjar, D. Spenser, and J. Duncan. Brain Shift Modeling for use in Neurosurgery. In MICCAI '98, pages 641-649, 1998. 202

4. S.F. Gibson. 3D Chainmail : a Fast Algorithm for Deforming Volumetric Objects. In Symposium on Interactive 3D Graphics, ACM SIGGRAPH, pages 149154, 1997. 203

5. M. Bro-Nielsen. Modeling Elasticity in Solids using Active Cubes: Application to Simulated Operations. In Computer Vision, Virtual Reality and Robotics in Medicine, pages 535-541, 1995. 203

6. S. Bauchemin and J.L. Barron. The Computation of Optical Flow. ACM computing surveys, 27(3), 1995. 203 
7. J. Dengler and M. Schmidt. The Dynamic Pyramid - a Model for Motion Analysis with Controlled Continuity. International Journal of Pattern Recognition and Artificial Intelligence, 2:275-288, 1988. 203

8. R. Bajcsy and S. Kovacic. Multi Resolution Elastic Matching. Computer Vision, Graphics and Image Processing, 46:1-21, 1989. 203

9. C. Davatzikos. Spatial Transformation and Registration of Brain Images using Elastically Deformable Models. Computer Vision and Image Understanding, 66(2):207-222, may 1997.203

10. G.E. Christensen, S.C. Joshi, and M.I. Miller. Volumetric Transformation of Brain Anatomy. IEEE Transactions on Medical Imaging, 16(6):864-877, december 1997. 203

11. M. Bro-Nielsen and C. Gramkow. Fast Fluid Registration of Medical Images. In Visualization in Biomedical Computing (VBC '96), pages 267-276, 1996. 203

12. D.K. Kyriacou and C. Davatzikos. A Biomechanical Model of Soft Tissue Deformation with Applications to Non-rigid Registration of Brain Images with Tumor Pathology. In MICCAI' '98, pages 531-538, 1998. 203

13. A. Hagemann, Rohr K., H.S. Stiel, U. Spetzger, and Gilsbach J.M. Non-Rigid Matching of Tomographic Images Based on a Biomechanical Model of the Human Head. In SPIE Medical Imaging '99, 1999. 203

14. O.C. Zienkewickz and R.L. Taylor. The Finite Element Method. McGraw Hill Book Co., 1987. 203, 204, 206

15. G.M. Nielson and J. Sung. Interval Volume Tetrahedrization. In Visualization '97, pages 221-228, 1997. 206

16. S. Balay, W.D. Gropp, L. Curfman McInnes, and B.F. Smith. PETSc 2.0 for MPI - Portable, Extensible Toolkit for Scientific Computations. http://www.mcs.anl.gov/petsc, 1998. 207

17. Will Schroeder, Ken Martin, and Bill Lorensen. The Visualization Toolkit: An Object-Oriented Approach to 3D Graphics. Prentice Hall PTR, New Jersey, 1996. 205, 207

18. J.L. Fleckenstein, J.V. Crues III, and C.D. Reimers. Muscle Imaging in Health and Disease. Springer, 1996. 208

19. B. Geiger. Three Dimensional Modeling of Human Organs and its application to diagnosis and surgical planning. Report 2105, INRIA Sophia-Antipolis France, 1993. 205 